\title{
Prediction of E-Learning Efficiency by Deep Learning in E- Khool Online Portal Networks
}

\author{
K. Srinivas \\ Professor, Department of Computer Science and Engineering, \\ Geethanjali College of Engineering and Technology, \\ (AUTONOMOUS) Cheeryal(V), Keesara(M), Medak(Dist).Telangana, INDIA. \\ katkamsrinu.01@gmail.com
}

\begin{abstract}
Nowadays, e-learning plays a very significant role in a global education system. E-learning is an effective way, which offers convenient and more benefits to users. In addition, it provides flexible learning and provides various contents based on the user's needs. However, identification of student's performance is a difficult one, because of virtual and dynamic environments. In this paper, Deep Belief Network (DBN) is used for predicting e-learning efficiency in e-khool online portal network. At first, key performance indicators, like the student's profile, student's behaviour data, student's performance, and teacher's involvement are determined for the prediction. Here, the student's performance, like name, age, family income, sex, and location, whereas the student's behaviour data, which includes the lecture hours, time spent on the discussion forum, attended exams, and a number of course subscribed are considered. Likewise, student's performance, such as average exam score and average time spent in the exam room, whereas teacher's involvement, such as the number of lectures given by teachers and the number of exams conducted by teachers are considered. Thus, the above-mentioned attributes are determined for each student. Then, the E-khool learning management system is introduced for extracting the performance indicators for further processing. Once the performance indicators are extracted, the deep learning model is applied for predicting the student performance using E-khool model. Here, the performance metrics, like Mean Absolute Error (MAE), accuracy, and Mean Square Error (MSE) are evaluated for analyzing the effectiveness of the developed method. Moreover, the DBN method obtains a maximum accuracy of $0.92 \%$ and also achieved a minimum MSE of $0.41 \%$ and a minimum MAE of $0.14 \%$.
\end{abstract}

Keywords: Deep Belief Network, E-Learning Efficiency, Deep Learning, Education System, Student's Performance.

\section{Introduction}

In recent times, the e-learning method has become more popular in the education system. The evaluation of e-learning efficiency helps to overcome the challenges faced in high school activities. Hence, the contributions to improve e-learning efficiency are widely considered in educational services [1]. Global education has two significant choices that are either to provide individuals with new approaches, knowledge, or skills by learning, and for changing the organizational location where individuals can learn. Nowadays, learning is performed by means of e-learning and also face to face ways. E-learning method is conducted anytime and everywhere, hence this method is a very attractive method for teachers and students [6].E-learning method is a hopeful solution for the learners to improve the capacities and skills by the fast development of the internet. Furthermore, it is very useful to educational organizations for improving their standard and offering learning content and tools [4]. E-learning is an application of training and studies in the education system and it also suitable for industrial resources [3]. Mainly, in the education system e-learning is encouraged, since the students are no need to come to the classroom. Moreover, learning schedules are flexible as well as transportation cost is avoided in the e-learning system [5]. In addition, the e-learning Recommender System (RS) provides flexibility to learners, less content searching time, offers suitable recommendations related to learners' interest, and increases the attention of learners [9] [7]. Even more, there are various filtering techniques, such as Hybrid Filtering (HF), Content-Based Filtering (CBF), and Collaborative Filtering (CF) to filter a learning content [7].

Normally, Neural Network (NN) is widely selected for e-learning efficiency prediction based on a balanced scorecard. NN is effectively utilized to model, analyze, predict and solve the exact problems in 
various methods, like management process, security systems, forecasting techniques, and so on [10] [11] [12] [1]. Generally, learning predictions contains several processes, like multimodal learning preprocessing and analyzing, learning feature selection, detection, and evaluation of learning [2]. Rather than machine learning techniques, such as association rules, logistic regression, and decision rules, NN is the promising method utilized in educational big data analysis [2]. Furthermore, various artificial intelligence methods, like fuzzy logic, NN, Bayesian classifiers, and hidden Markov models are utilized for e-learning efficiency prediction. The intelligent e-learning system was developed in [13] [8], and it automates a teaching method in e-learning. In addition, Gravitational Search-based Back Propagation Neural Network (GSBPNN) technique was introduced for predicting the learning styles of the learner in real time. Similarly, data mining methods were also applied for extracting the knowledge from the elearning system through the users. In [14] [3], a bipartite network was developed, and it includes domain knowledge by topological properties in a recommendation system. Here, the domain connection among product weights, weight, and recommendation to the customer are computed for improving quality of recommendation. The naive-Bayes tree method was applied as a classification approach for categorizing the learning styles of learners using the Felder-Silverman Learning Styles Model (FSLSM) [15] [4]. The fuzzy test and discriminant index approach was introduced for recognizing service items, which also required Performance Evaluation Matrix (PEM) enhancement [16] [5].

The main intention of the research is the e-learning efficiency prediction in e-khool online portal network using DBN. Initially, the key performance indicators, such as student's profile, student's behaviour data, student's performance, and teacher's involvement are determined for e-learning efficiency prediction. Here, each performance indicator has its functions. After that, e-khool learning management system is introduced for extracting the performance indicators. Finally, DBN is developed to predict the performance of students using e-khool model.

\section{The major contribution of the paper:}

DBN for e-learning efficiency prediction in e-khool online portal network: The classifier, named DBN is used for predicting e-learning efficiency in e-khool online portal network effectively. The key performance indicators are initially identified, and then extract performance indicators using E-Khool learning management system. Finally, the DBN is utilized for predicting the e-learning efficiency with improved performance.

The paper is arranged as follows: section 2 elaborates literature review of existing e-learning efficiency prediction methods using a deep learning network. Section 3 discussed the developed deep learning technique for student performance prediction in E-khool models. Section 4 illustrates the results and discussion of the proposed method and in the last, section 5 concludes the paper.

\section{Motivation}

This section explains the motivation of the e-learning efficiency method in the e-khool online portal network. The drawbacks of existing e-learning efficiency techniques are considered to create a novel prediction technique for e-learning efficiency in e-khool online portal network.

\subsection{Literature Survey}

This sub-section elaborates the existing e-learning efficiency prediction approaches. Halachev P [1] developed an NN-based balance scorecard method for e-learning prediction. Initially, the input data was taken and it was pre-processed for further processing. The pre-processing process was employed by Principal Component Analysis (PCA) and interpolation methods. The number of variables and sample size were decreased by the pre-processing process. After that, the linear NN and non-linear NN were employed for optimizing structures. In addition, the Optimal Brain Damage (OBD) method was also applied in non-linear NNs for attaining high accuracy of e-learning prediction. However, this method was not developed for predicting e-learning efficiency in higher education. Wang Xet al. [2] introduced a convolutional gated recurrent unit and $\mathrm{NNs}$ to predict the e-learning performance of students. The raw data was selected as the input data from e-learning systems. At first, a general learning feature quantification technique was developed for converting the raw input data into groups of independent learning characteristics. Furthermore, the weighted average pooling method is selected in the convolutional gated recurrent unit for the prediction of learning performance. Consequently, enhanced parallel explainable $\mathrm{NN}$ was also employed for clarifying the prediction outcome. However, the developed method was failed to gather more effective datasets for improving prediction accuracy. BhamaPRet al. [3] presented a user's domain knowledge prediction in an e-learning system. The fuzzy cognitive map was employed for finding the dependencies and also finds user knowledge in other fields. Moreover, virtual reality and augmented reality were also utilized for providing an interactive learning experience to 
users. Here, user commands were accepted as audio signals and are categorized into system commands to create the response. Furthermore, discrete wavelet transforms and wavelet packet decomposition was employed for feature extraction. Consequently, the Artificial Neural Network (ANN) was also utilized for the classification process. Although, this method was not avoided the usage of gadgets in the entire system for enhancing system security. El AissaouiO et al. [4] developed a fuzzy classification technique for learning predictions in an e-environment. Here, a generic technique was applied for identifying the learning styles. Generally, this developed method includes major two stages, namely the extraction of learning sequences and classification stage. At first, the learning sequences were extracted from learner's log files by web usage mining approaches. After that, extracted learner sequences were classified based on a particular learning style method and clustering approaches. In addition, the Felder-Silverman method and fuzzy C-means clustering technique were applied for executing e-learning prediction. In this method, the time complexity and computation cost were highly reduced, but still, this method was not applied to other machine learning techniques for improving accuracy.

\subsection{Challenges}

The challenges faced by present e-learning efficiency prediction are elaborated below:

- The convolutional gated recurrent unit and explainable NNs were developed in [2] for predicting students learning performance in the e-learning environment. However, the developed NN method was not applied for additional learning features, such as learning path generating, trend prediction, and sentiment analysis.

- In [3], the user's domain knowledge prediction in e-learning was introduced, but still, the developed method was failed to group users on the internet for enhancing activity-based learning.

- The fuzzy classification technique [4] was utilized for e-learning prediction. Although this method was not considered other machine learning methods for enhancing the prediction accuracy.

- The hybrid e-learning recommendation approach [7] was presented for e-learning prediction. On the other hand, this method was not included more characteristics of learners for improving the prediction.

- In [2], conventional gated recurrent unit and explainable NNs was developed for e-learning prediction. However, the developed method was not included in early prediction techniques in real-life e-learning systems.

\section{Identifying Key Performance Indicators for Prediction}

In this section, the performance indicators, such as student profile, student behaviour data, student performance, and teacher's involvement are explained.

\section{a) Student profile}

In the student profile performance indicator, student's personal details are considered for e-learning efficiency prediction. Here, student's personal details, like name, age, sex, family income, and location are determined for each student for the prediction, and is represented as $\mathrm{k}_{1}, \mathrm{k}_{2}, \mathrm{k}_{3}, \mathrm{k}_{4}$ and $\mathrm{k}_{5}$ respectively.

\section{b) Student behavior data}

In the student behaviour data, the number of hours spent for a lecture, average time spent on the discussion forum, number of exams attended, and number of course subscribed are identified for each student, and the student behaviour data attributes are denoted as $\mathrm{k}_{6}, \mathrm{k}_{7}, \mathrm{k}_{8}$ and $\mathrm{k}_{9}$.

\section{c) Student performance}

The next key performance indicator is student performance. Here, the average exam score and average time spent in the exam room are identified for each student and is represented as $\mathrm{k}_{10}$ and $\mathrm{k}_{11}$.

\section{d) Teacher's involvement}

Teacher's involvement is another key performance indicator for e-learning efficiency prediction. Number of lectures given by teachers and the number of exams conducted by teachers are the two performance indicators in the teacher's involvement. These two indicators are represented as $k_{12}$ and $k_{13}$.

The key performance vector is expressed as,

$$
\mathrm{K}=\left\{\mathrm{k}_{1}, \mathrm{k}_{2}, \mathrm{k}_{3}, \ldots \mathrm{k}_{13}\right\}
$$

\subsection{E-khool Learning Management System to Extract Performance Indicators}

Generally, the e-khool learning management system [20] consists of four significant units, like the lecturer unit, student unit, information unit, and interface unit. In the lecturer unit, the analysis of 
competencies, assessment of exams, valuation of Visual, Aural, Read/write, and Kinesthetic sensory modalities (VARK) survey and selector representative of learning method is performed. Normally, the assessment of exams is utilized for identifying the knowledge of students. In addition, the status of knowledge is also utilized for analyzing the competencies in the lecturer unit. Moreover, the evaluation of VARK survey and selector representative of the learning method decides about the training and learning methods of the student. After the lecture unit, the status of knowledge and learning and training methods of students is checked in the student unit. The third unit is the information unit where the competencies, learning method, and training schemes of subjects and status of knowledge are included in the education contents. Then, the education contents, and the learning method and training schemes of student are given to interface representative in the interface unit to illustrate the contents. Moreover, the director of competencies, VARK survey, director of the learning method, and the training schemes, configuration of contents, and the supervisor of exams are included in the interface unit. In addition, the supervisor of exams shows the status of knowledge for every student. Furthermore, learning and training schemes of students in the student unit and educational contents in the information unit are given to interface representatives in the interface unit for illustrating contents. The systematic diagram of ekhool learning management system is depicted in Fig. 1.

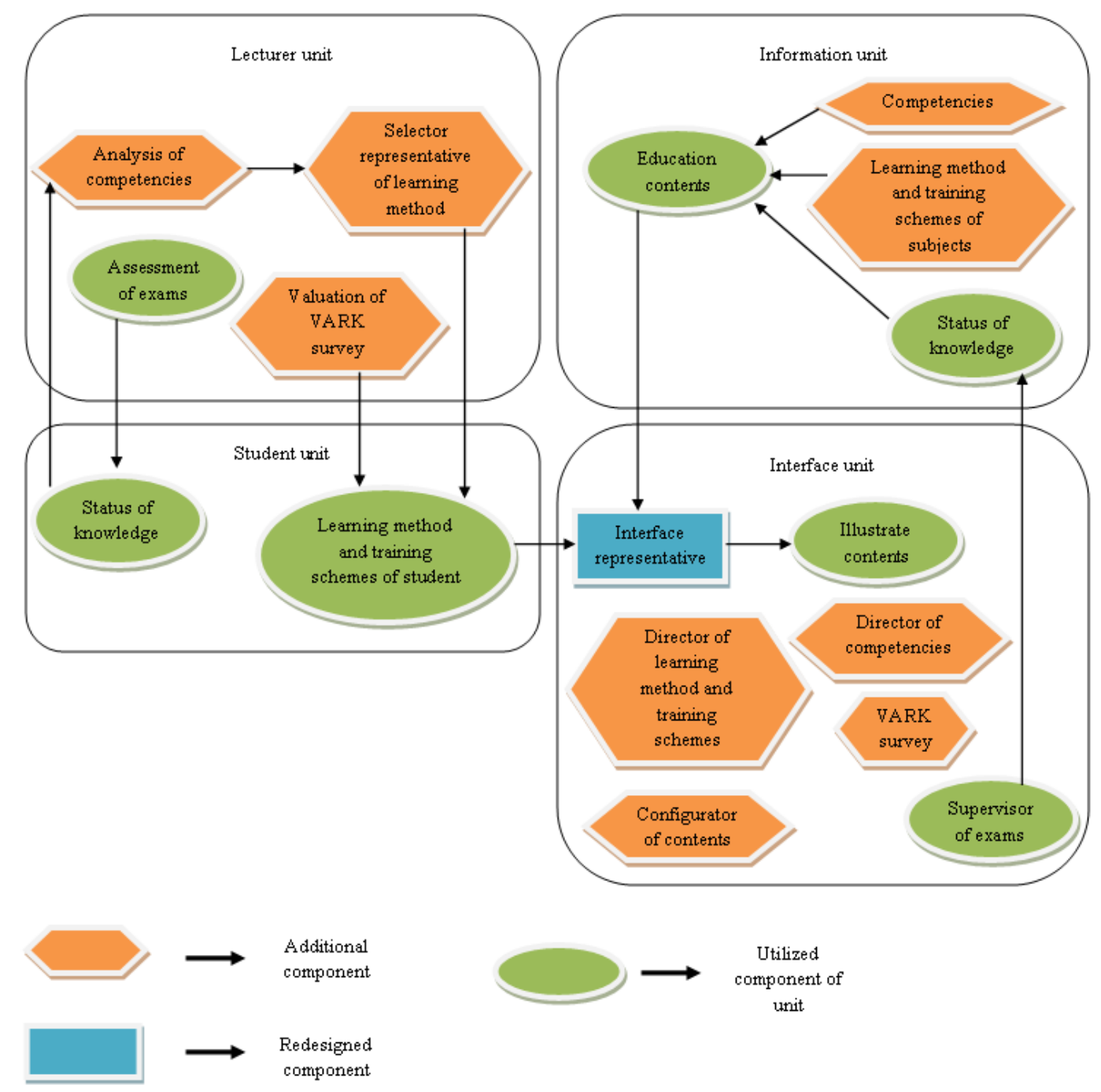

Fig. 1.Architecture of E-khool learning management system

\subsection{Deep Learning Model for Predicting the Student Performance Using E-Khool Model}

The DBN [17] is used to predict the e-learning performance of students using the e-khool network. The total key performance indicator $K$ is taken as input to the DBN method. The DBN architecture is the type of Deep Neural Network (Deep NN) [19], which includes Restricted Boltzmann Machine (RBM) layers and multilayer perceptron (MLP) layers that are linked with weights. The MLP layer includes an 
input layer, output layer, and hidden layer, and also RBM layer includes a hidden layer and visible layer. Furthermore, visible layers are fully interconnected with hidden layers. The characteristics of DBN have no connections among hidden layers and visible layers. In addition, interconnections are symmetric and restricted among visible layers and hidden layers. Here, the DBN method is considered to predict the elearning efficiency in e-khool online portal network. The architecture of DBN is signified in Fig. 2.

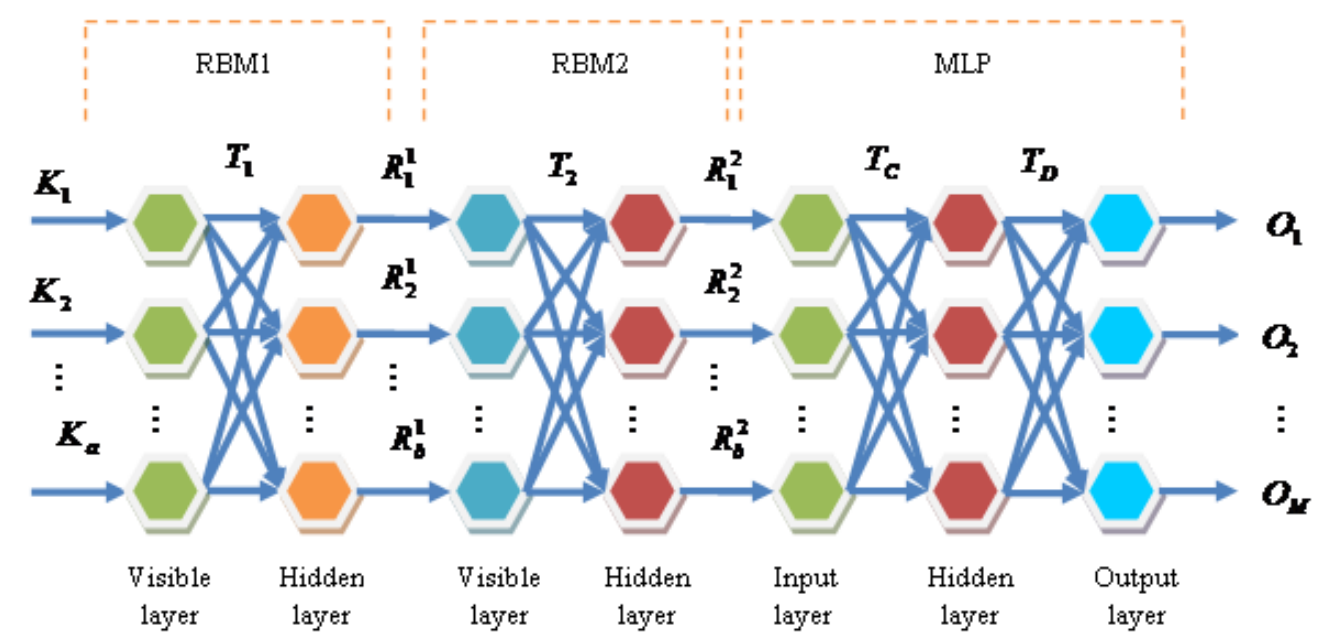

Fig.2. The architecture of the DBN classifier

The performance indicators are taken as input and given to the visible layer and the hidden layer of $\mathrm{RBM} 1$ is represented as,

$$
\begin{aligned}
& \mathrm{K}^{1}=\left\{\mathrm{K}_{1}^{1}, \mathrm{~K}_{2}^{1}, \ldots, \mathrm{K}_{\mathrm{u}}^{1}, \ldots \mathrm{K}_{\mathrm{a}}^{1}\right\} ; 1 \leq \mathrm{u} \leq \mathrm{a} \\
& \mathrm{R}^{1}=\left\{\mathrm{R}_{1}^{1}, \mathrm{R}_{2}^{1}, \ldots, \mathrm{R}_{\mathrm{v}}^{1}, \ldots \mathrm{R}_{\mathrm{b}}^{1}\right\} ; 1 \leq \mathrm{v} \leq \mathrm{b}
\end{aligned}
$$

where, the $\mathrm{u}^{\text {th }}$ visible neuron of RBM 1 is represented as $K_{a}^{1}$, the term $R_{v}^{1}$ denotes to $v^{\text {th }}$ hidden neuron, the total hidden neuron is characterized by $\mathrm{b}$. In which, the visible layer and hidden layer consists of neurons, where each neuron creates bias. Let us assume Q and $R$ specifies the biases in visible layer and hidden layer and RBM 1 layer biases is represented as,

$$
\begin{aligned}
& \mathrm{Q}^{1}=\left\{\mathrm{Q}_{1}^{1}, \mathrm{Q}_{2}^{1}, \ldots \mathrm{Q}_{\mathrm{u}}^{1}, \ldots \mathrm{Q}_{\mathrm{a}}^{1}\right\} \\
& \mathrm{R}^{1}=\mathrm{R}_{1}^{1}, \mathbf{R}_{2}^{1}, \ldots, \mathbf{R}_{\mathrm{v}}^{1}, \ldots \mathbf{R}_{\mathrm{b}}^{1}
\end{aligned}
$$

where, the term $Q_{u}^{1}$ denotes bias of $u^{\text {th }}$ visible neuron, and $R_{v}^{1}$ signifies bias of $v^{\text {th }}$ hidden neuron. The weight vector for RBM 1is specified by,

$$
\mathrm{T}^{1}=\left\{\mathrm{T}_{\mathrm{u}, \mathrm{v}}^{1}\right\} ; 1 \leq \mathrm{u} \leq \mathrm{a} ; 1 \leq \mathrm{v} \leq \mathrm{b}
$$

where, weight between $\mathrm{U}^{\text {th }}$ visible neuron and $\mathrm{V}^{\text {th }}$ hidden neuron is expressed by $\mathrm{T}_{\mathrm{u}, \mathrm{v}}^{1}$. Here, hidden layer output from RBM1 is estimated by means of bias and weights associated to each visible neuron and represented by,

$$
\mathrm{H}_{\mathrm{v}}^{1}=\gamma\left[\mathrm{R}_{\mathrm{v}}^{1}+\sum_{\mathrm{T}}\left(\mathrm{K}_{\mathrm{u}}^{\mathrm{v}}\right) \mathrm{T}_{\mathrm{u}, \mathrm{v}}^{1}\right]
$$

where, the term $\gamma$ signifies activation function. Consequently, the output attained from RBM1 is represented by,

$$
\mathrm{H}^{1}=\left\{\mathrm{H}_{\mathrm{v}}^{1}\right\} ; 1 \leq \mathrm{v} \leq \mathrm{b}
$$

Afterwards, RBM1output is given as the input to the RBM 2 visible layer. Therefore, RBM2layer input is signified by $\mathrm{K}^{2}$. Similarly, RBM 2 hidden layer is designated as $\mathrm{R}^{2}$. The RBM2 weight vector is indicated as $\mathrm{T}^{2}$ and $\mathrm{v}^{\text {th }}$ hidden neuron output is denoted by $\mathrm{R}_{\mathrm{v}}^{2}$ and bias is associated with $\mathrm{v}^{\text {th }}$ hidden neuron is $R_{v}^{2}$. Thus, the result produced by the hidden layer is expressed by $R^{2}$.

The RBM 2 hidden layers output is subjected to the MLP input layer. Therefore, input layer MLP is denoted by,

$$
\mathrm{I}=\left\{\mathrm{I}_{1}, \mathrm{I}_{2}, \ldots, \mathrm{I}_{\mathrm{v}}, \ldots \mathrm{I}_{\mathrm{e}}\right\}=\left\{\mathrm{H}_{\mathrm{v}}^{2}\right\} ; 1 \leq \mathrm{v} \leq \mathrm{b}
$$

where, the term $\mathrm{v}$ signifies the total neuron in the input layer. The expression of hidden layer MLP is specified by, 
Prediction of E-Learning Efficiency by Deep Learning in E-Khool Online Portal Networks

$$
\mathrm{M}=\left\{\mathrm{M}_{1}, \mathrm{M}_{2}, \ldots \mathrm{M}_{\mathrm{X}}, \ldots, \mathrm{M}_{\mathrm{Y}}\right\} ; 1 \leq \mathrm{X} \leq \mathrm{Y}
$$

where, Y denotes total hidden neurons, let us assume $\mathrm{b}_{\mathrm{d}}$ be the bias of hidden neuron $X$, where $\mathrm{X}=1,2, \ldots, \mathrm{Y}$. The MLPs output layer is denoted by,

$$
\mathrm{X}=\left\{\mathrm{X}_{1}, \mathrm{X}_{2}, \ldots \mathrm{X}_{\mathrm{O}}, \ldots \mathrm{X}_{\mathrm{P}}\right\} ; 1 \leq \mathrm{O} \leq \mathrm{P}
$$

where, P denotes whole neurons in the output layer. Here, MLP has taken two weight vectors; one is offered between the hidden and input layer and the other existed among output and hidden layer. Let us consider $\mathrm{T}^{\theta}$ be weight vector among hidden and input layers, and is demonstrated by,

$$
\mathrm{T}^{\theta}=\left\{\mathrm{T}_{\mathrm{vX}}^{\beta}\right\} ; 1 \leq \mathrm{v} \leq \mathrm{b} ; 1 \leq \mathrm{X} \leq \mathrm{Y}
$$

where, $\mathrm{T}_{\mathrm{vX}}^{\beta}$ demotes to weight among neuron $\mathrm{v}$ and hidden neuron $\mathrm{X}$. The hidden layer output is demonsrated as,

$$
\mathrm{M}_{\mathrm{v}}=\left[\sum_{\mathrm{v}=1}^{\mathrm{b}} \mathrm{T}_{\mathrm{v}, \mathrm{X}}^{\delta} * \mathrm{M}_{\mathrm{X}}\right] \delta_{\mathrm{X}}
$$

where, term $\mathrm{M}_{\mathrm{X}}$ denotes the input layer $\mathrm{X}$ of MLP. The weights among the hidden and output layer are given by $\mathrm{T}^{\alpha}$ and are illustrated as,

$$
\mathrm{T}^{\phi}=\left\{\mathrm{T}_{\mathrm{XO}}^{\phi}\right\} ; 1 \leq \mathrm{X} \leq \mathrm{Y} ; 1 \leq \mathrm{O} \leq \mathrm{P}
$$

Consequently, the output vector is calculated by weights $\mathrm{T}^{\phi}$ and hidden layer output is expressed by,

$$
\mathrm{E}_{\mathrm{w}}=\sum_{\mathrm{X}=1}^{\mathrm{Y}} \mathrm{T}_{\mathrm{XO}}^{\phi} * \mathrm{M}_{\mathrm{v}}
$$

where, $\mathrm{T}_{\mathrm{XO}}^{\phi}$ denotes weight between the hidden neuron $\mathrm{X}$ and output neuron $\mathrm{O}$ and hidden layer output is represented as $\mathrm{M}_{\mathrm{v}}$.

\section{Results and Discussion}

The results and discussion of a developed method are illustrated in this section. The experimental setup, performance metrics, competing methods, and comparative analysis are given below.

\subsection{Experimental Setup}

The execution of the developed e-learning efficiency prediction method is executed using PYTHON with 4GB RAM, Windows 8 OS with Intel core i-3 processor.

\subsection{Dataset Description}

The experimentation of the proposed DBN for e-learning efficiency prediction is done based on the data collected from the E-Khool platform, which carries the information of student's information's that includes the name, age, location, average exam score, average time spent in the exam room, and so on.

\subsection{Performance Metrics}

The performance of DBN is evaluated by three performance metrics, accuracy, MSE, and MAE.

a) Accuracy: It is computed as the degree of closeness of approximated value regarding its original, also it is expressed as,

$$
A c c=\frac{A_{o}+A_{e}}{A_{o}+R_{e}+A_{e}+R_{o}}
$$

b) MSE: The MSE is estimated by the below expression,

$$
\mathrm{MSE}=\frac{1}{\mathrm{P}} \sum_{\alpha=1}^{\mathrm{P}}\left(\mathrm{E}_{\alpha}-\hat{\mathrm{E}}_{\alpha}\right)^{2}
$$

where, $\mathrm{P}$ is the predictors and $\left(\mathrm{E}_{\alpha}-\hat{\mathrm{E}}_{\alpha}\right)^{2}$ illustrates the square of error.

c) MAE: The MAE is calculated to determine the average error in a prediction set, with no direction. The MAE is represented as, 


$$
\mathrm{MAE}=\frac{1}{\mathrm{Z}} \sum_{\mathrm{r}=1}^{\mathrm{Z}}\left|\mathrm{b}_{\mathrm{r}}-\hat{\mathrm{b}}_{\mathrm{r}}\right|
$$

where, $\mathrm{Z}$ denotes total items, $b_{\mathrm{r}}$ describes real profusion fraction for $\mathrm{r}^{\text {th }}$ end member, and $\hat{b}_{\mathrm{r}}$ specifies the calculated value.

\subsection{Competing Methods}

The techniques, such as deep learning, NN [19], Ride Neural Network (Ride NN) [18], and Bayesian classifier are considered for evaluating the efficiency of developed DBN.

\subsection{Comparative Analysis}

The comparative analysis of proposed DBN with regards to accuracy, MSE, and MAE parameters are estimated by changing training data percentage using 100 learners based on course 1 , course 2 , and course 3 .

\subsubsection{Analysis using course-1}

The performance analysis with respect to the accuracy, MAE, and MSE by changing the training data using course 1 is portrayed in Fig. 3. The analysis in terms of accuracy parameter is represented in Fig. 3a). For 90\% training data, accuracy value estimated through present deep learning, NN, Ride NN, Bayesian classifier, and DBN is $0.87,0.88,0.89,0.91$, and 0.95 , respectively. The analysis of methods using the MSE parameter is specified in Fig. 3b). For 90\% of training data, MSE value obtained by existing deep learning, NN, Ride NN, Bayesian classifier, and DBN are 0.65, 0.62, 0.58, 0.54, and 0.50\%. The analysis of various techniques based on the MAE parameter is shown in Fig. 3c). When training data $=90 \%$, MAE value computed by existing deep learning, NN, Ride NN, Bayesian classifier, and DBN are $0.37,0.35,0.34,0.29$, and 0.24 .

\subsubsection{Analysis using course-2}

The performance analysis with respect to the accuracy, MAE, and MSE by varying training data percentage based on course- 1 is displayed in Fig. 4. The analysis using the accuracy parameter is plotted in Fig. 4a). For $90 \%$ of training data, accuracy value estimated through present deep learning, NN, Ride NN, Bayesian classifier, and DBN are 0.88, 0.91, 0.92, 0.92, and 0.93, respectively. The analysis of methods with regards to the MSE parameter is represented in Fig. 4b). When $90 \%$ of training data is considered, MSE value obtained by existing deep learning, NN, Ride NN, Bayesian classifier, and DBN are $0.60,0.58,0.57,0.49$, and 0.46 . The analysis of methods in terms of MAE parameter is showed in Fig. 4c). When training data $=90 \%$, MAE value evaluated by existing deep learning, NN, Ride NN, Bayesian classifier and DBN are $0.35,0.33,0.31,0.25$, and 0.20 .

\subsubsection{Analysis based on course-3}

The performance analysis with respect to the accuracy, MAE, and MSE by changing the training data using course- 1 is represented in Fig. 5. The analysis in terms of accuracy parameter is represented in Fig. 5a). For $90 \%$ of training data, accuracy value estimated through existing deep learning, NN, Ride NN, Bayesian classifier, and DBN is $0.89,0.91,0.91,0.91$, and 0.93 , respectively. The analysis of methods regards to MSE parameter is specified in Fig. 5b). For 90\% of training data, MSE value obtained by existing deep learning, NN, Ride NN, Bayesian classifier, and DBN are 0.53, 0.50, 0.48, 0.45, and 0.40. The analysis of various techniques using MAE parameter is portrayed in Fig. 5c). When training data is $90 \%$, MAE value computed by existing deep learning, NN, Ride NN, Bayesian classifier, and DBN is $0.20,0.19,0.19,0.17$, and 0.15 . 


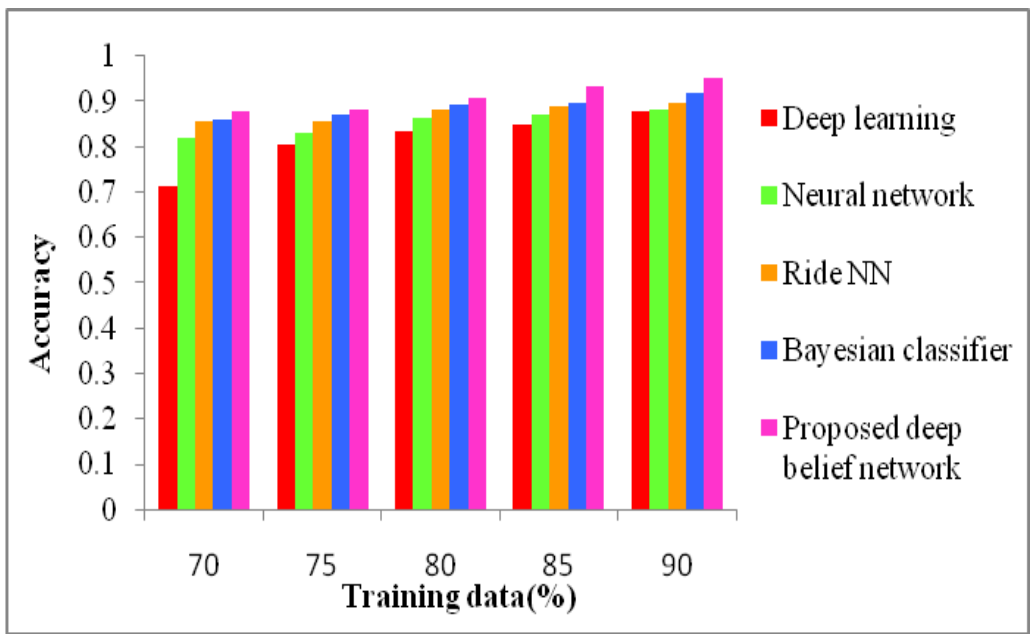

(a)

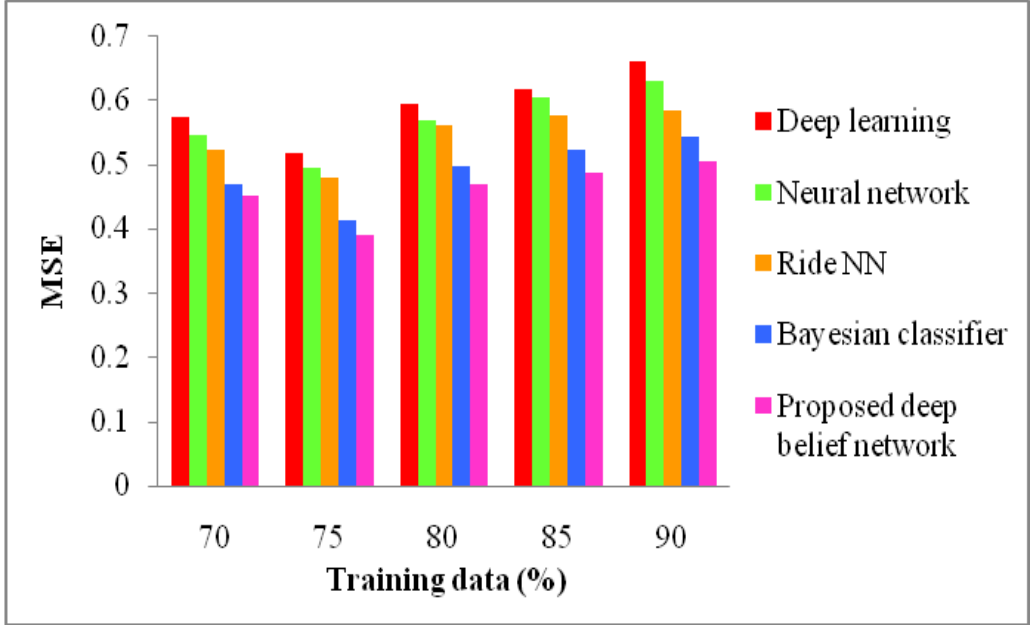

(b)

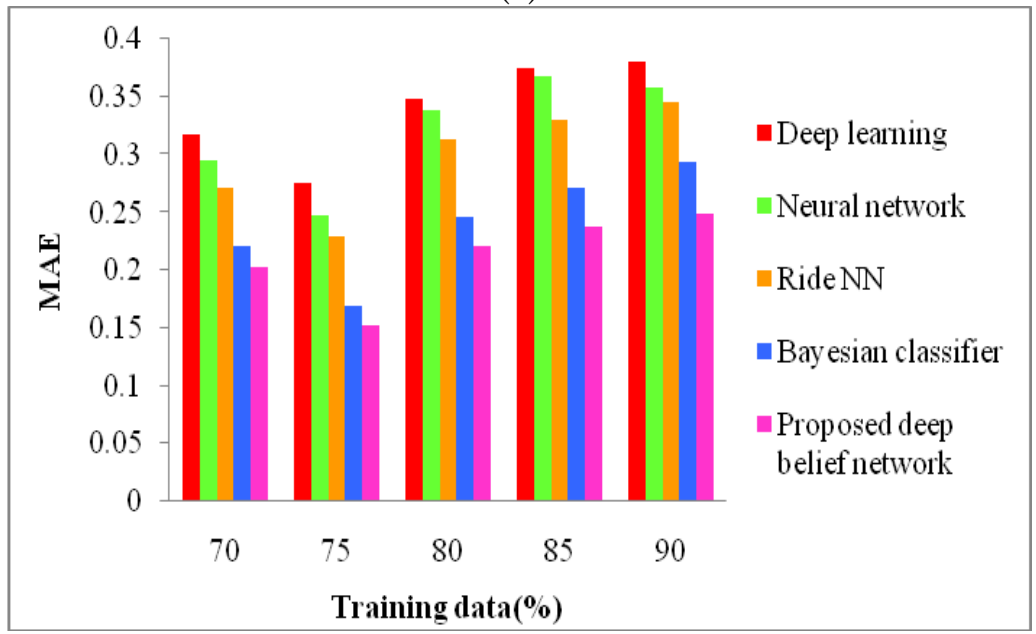

(c)

Fig.3. Analysis based on course-1in terms of a) Accuracy, b) MSE, c) MAE 
Prediction of E-Learning Efficiency by Deep Learning in E-Khool Online Portal Networks

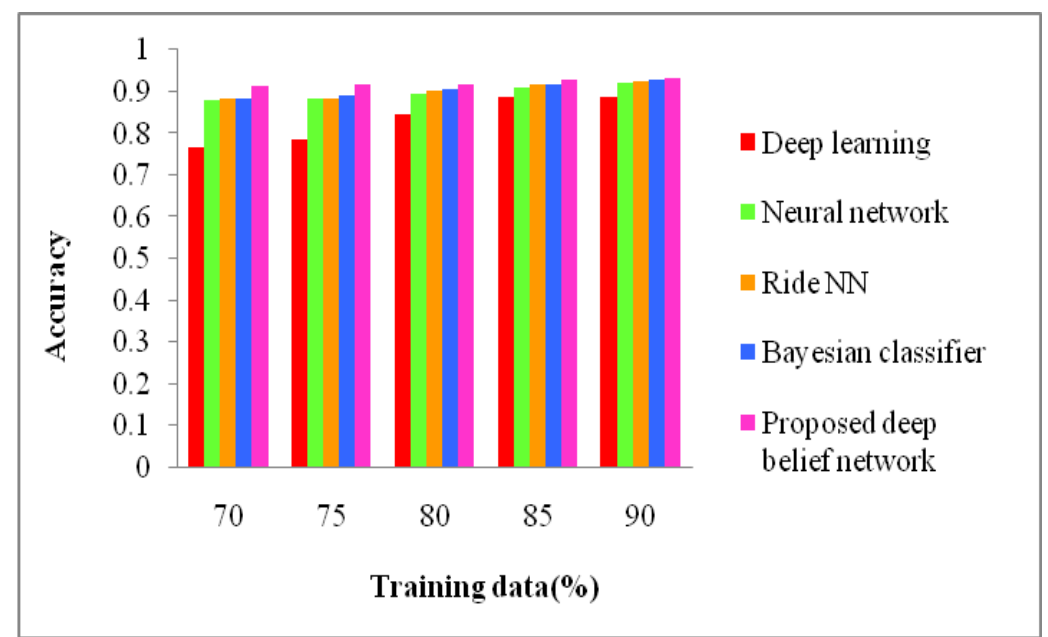

(a)

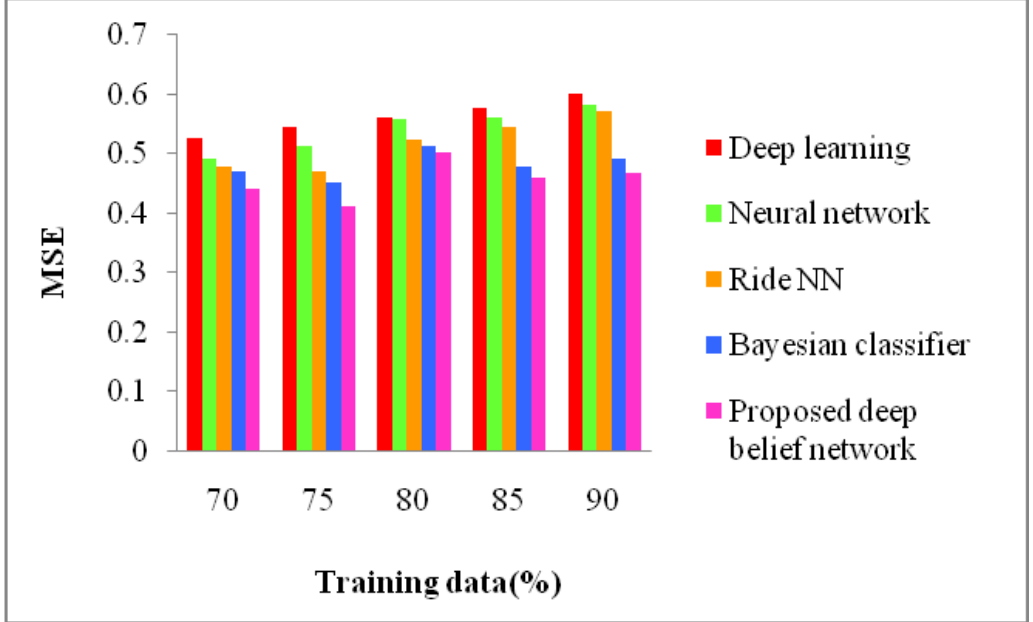

(b)

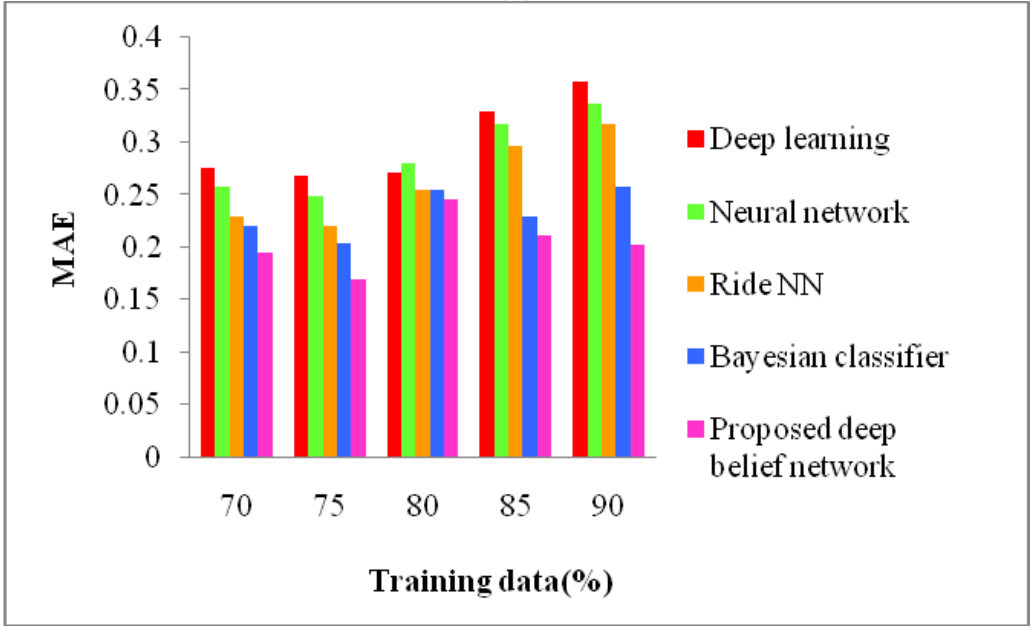

(c)

Fig.4. Analysis based on course-2 using a) Accuracy, b) MSE, c) $M A E$ 


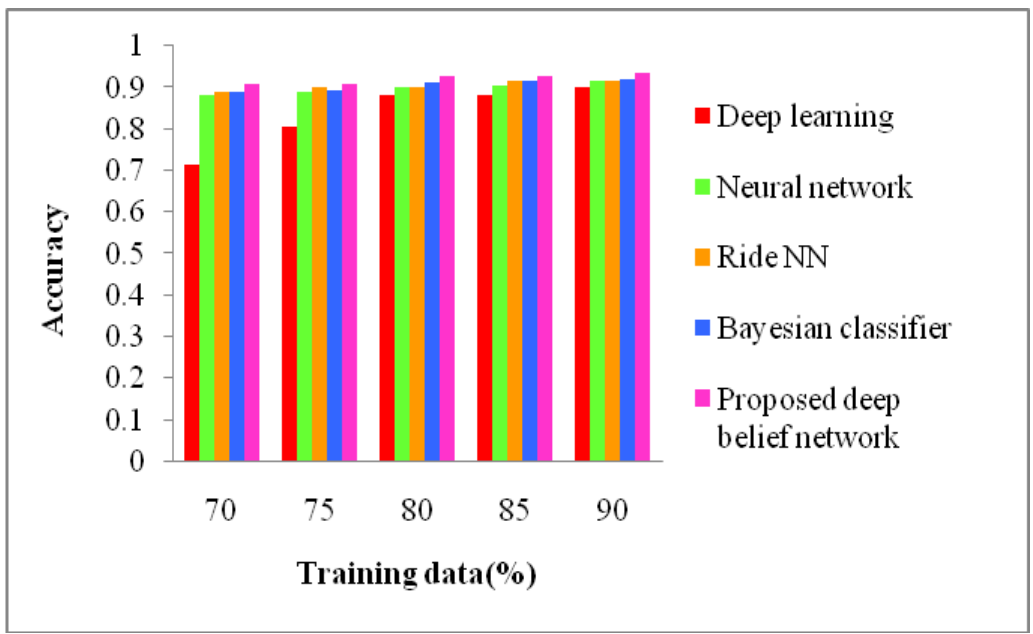

(a)

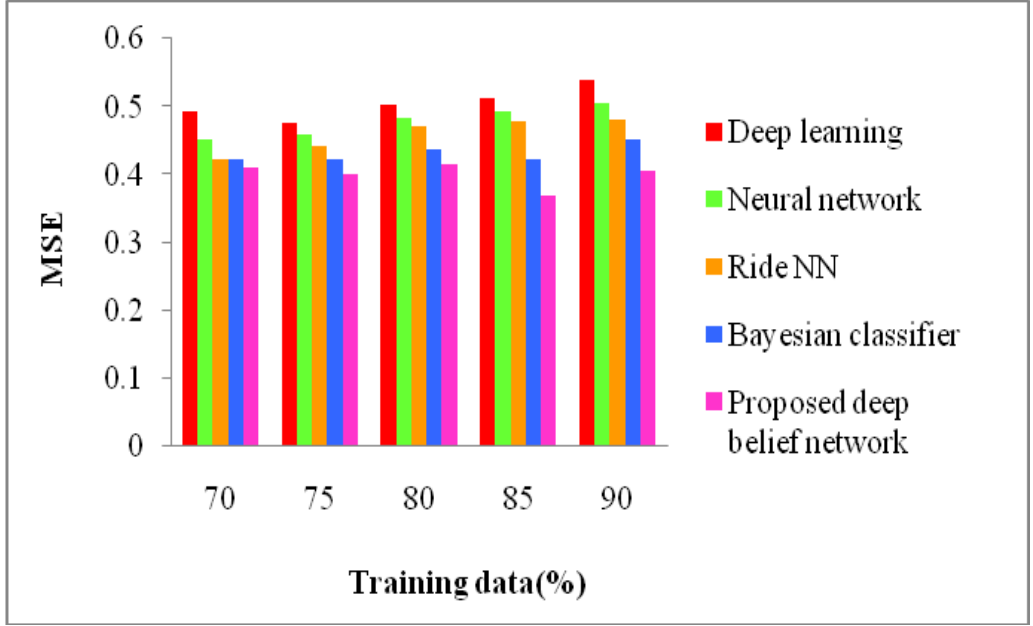

(b)

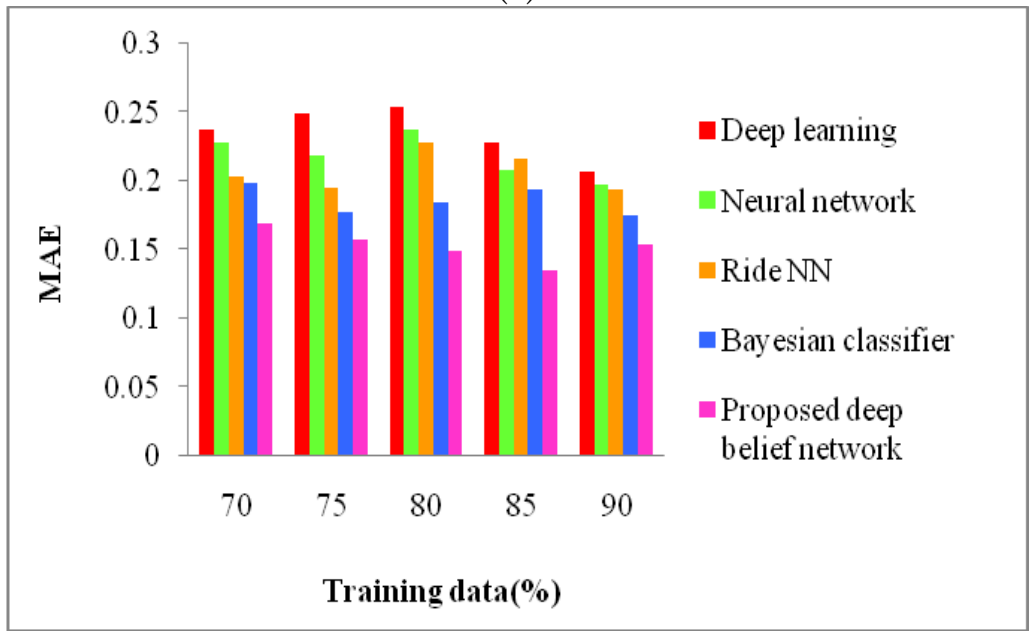

(c)

Fig.5. Analysis based on course-3 based on a) Accuracy, b) MSE, c) MAE 


\subsection{Comparative Discussion}

The analysis of different methods in terms of training data using course-1, course-2, and course-3using accuracy, MSE, and MAE parameter is represented in table 1. The maximum accuracy of 0.93 is obtained by developed DBN, while the existing techniques, such as deep learning, NN, Ride NN, Bayesian classifier attained an accuracy of $0.84,0.86,0.88$, and 0.89 using course- 1 for $85 \%$ of training data. Furthermore, a minimum MSE value of 0.48 is attained by developed DBN for training data $=85 \%$ using course-1. Likewise, the developed DBN obtained a minimum MAE value of 0.23 based on course- 1 using 85 training data. From the below comparative analysis table, it is noted that developed DBN has maximum accuracy, minimum MSE, and MAE.

Table1. Comparative Analysis

\begin{tabular}{|c|c|c|c|c|c|c|}
\hline $\begin{array}{c}\text { Course } \\
\text { type }\end{array}$ & Metrics & $\begin{array}{c}\text { Deep } \\
\text { learning }\end{array}$ & NN & Ride NN & $\begin{array}{l}\text { Bayesian } \\
\text { classifier }\end{array}$ & Proposed DBN \\
\hline \multirow[t]{3}{*}{ Course-1 } & Accuracy & 0.83 & 0.86 & 0.88 & 0.89 & 0.90 \\
\hline & $M S E$ & 0.59 & 0.56 & 0.55 & 0.49 & 0.46 \\
\hline & $M A E$ & 0.34 & 0.33 & 0.31 & 0.24 & 0.22 \\
\hline \multirow[t]{3}{*}{ Course-2 } & Accuracy & 0.84 & 0.89 & 0.90 & 0.90 & 0.91 \\
\hline & $M S E$ & 0.56 & 0.55 & 0.52 & 0.51 & 0.50 \\
\hline & $M A E$ & 0.27 & 0.27 & 0.25 & 0.25 & 0.24 \\
\hline \multirow[t]{3}{*}{ Course-3 } & Accuracy & 0.88 & 0.89 & 0.90 & 0.91 & 0.92 \\
\hline & $M S E$ & 0.50 & 0.48 & 0.47 & 0.43 & 0.41 \\
\hline & $M A E$ & 0.25 & 0.23 & 0.22 & 0.18 & 0.14 \\
\hline
\end{tabular}

\section{Conclusion}

In this research paper, the DBN is used to predict the effectiveness of e-learning in e-khool online portal network. Initially, key performance indicators, namely student's profile, student's behaviour data, student's performance, and teacher's involvement are estimated for e-learning efficiency prediction. Here, name, age, family income, sex, location, lecture hours, time spent on the discussion forum, attended exams number of course subscribed, average exam score, average time spent on exam room, number of lectures given by teachers, and number of exams conducted by teachers are identified. Once the key performance is determined, the e-khool learning management system is employed to extract the accurate performance indicators for further processing. Then, the extracted performance indicators are subjected to a DBN system for predicting the e-learning efficiency of students. In addition, performance metrics, such as MAE, accuracy, and MSE are considered for analyzing the effectiveness of the method. Consequently, DBN is used for identifying the performance of student's performance in e-khool system. The DBN method obtains maximum accuracy of 0.92 , minimum MSE of 0.41, and minimum MAE of 0.14. In the future, we will develop a new classifier to test the effectiveness of the e-learning in e-khool online portal network.

\section{Compliance with Ethical Standards}

Conflicts of interest: Authors declared that they have no conflict of interest.

Human participants: The conducted research follows the ethical standards and the authors ensured that they have not conducted any studies with human participants or animals.

\section{References}

[1] Halachev P, "Prediction of E-Learning efficiency by neural networks", Cybernetics and information technologies, vol.12, no.2, pp.98-108, June 2012.

[2] Wang X, Wu P, Liu G, Huang Q, Hu X, Xu H, "Learning performance prediction via convolutional GRU and explainable neural networks in e-learning environments”, Computing, vol.101, no.6, pp.587-604, June 2019.

[3] Bhama PR, Hariharasubramanian V, Mythili O P, Ramachandran M, "Users' domain knowledge prediction in elearning with speech-interfaced augmented and virtual reality contents", Virtual Reality, vol.24, no.1, pp.163-73, March 2020.

[4] El Aissaoui O, El Madani Y E, Oughdir L, El Allioui Y, “A fuzzy classification approach for learning style prediction based on web mining technique in e-learning environments", Education and Information Technologies, vol.24, no.3, pp.1943-59, May 2019. 
[5] Lee TS, Wang CH, Yu C M, "Fuzzy Evaluation Model for Enhancing E-Learning Systems", Mathematics, vol.7, no.10, pp.918, October 2019.

[6] Siagian S, Sinambela P N, Wau Y, "Effectiveness and Efficiency of E-learning in Instructional Design", World Transactions on Engineering and Technology Education, vol.18, no.1, pp.73-7, 2020.

[7] Wan S and Niu Z, "A hybrid e-learning recommendation approach based on learners' influence propagation" IEEE Transactions on Knowledge and Data Engineering, vol.32, no.5, pp.827-40, January 2019.

[8] Blagojević M and Micić Ž, "A web-based intelligent report e-learning system using data mining techniques", Computers \& Electrical Engineering, vol.39, no.2, pp.465-74, February 2013.

[9] Bourkoukou O, El Bachari E, El Adnani M, "A recommender model in e-learning environment", Arabian Journal for Science and Engineering, vol.42, no.2, pp.607-17, February 2017.

[10] Moody J, "Economic forecasting: Challenges and neural network solutions" InProceedings of the international symposium on artificial neural networks, pp.789-800, December 1995.

[11] Hou Z E and Duan F J, "The Neural Network Method of Economy Forecasting", In proceedings of 2009 WRI World Congress on Software Engineering, vol.3, pp.95-97, May 2009.

[12] Maciel L S and Ballini R, "Design a neural network for time series financial forecasting: Accuracy and robustness analysis", Anales do $9^{\circ}$ EncontroBrasileiro de Finanças, Sao Pablo, Brazil, 2008.

[13] Prakasam S, "An agent-based intelligent system to enhance e-learning through mining techniques", In proceedings of International Journal on Computer Science and Engineering, 2010.

[14] Li J, Zhang L, Meng F, Li F, "Recommendation algorithm based on link prediction and domain knowledge in retail transactions" Procedia Computer Science, vol.31, pp.875-81, January 2014.

[15] Abdullah M, Daffa WH, Bashmail R M, Alzahrani M, Sadik M, "The impact of learning styles on Learner's performance in E-learning environment", International Journal of Advanced Computer Science and Applications, vol.6, no.9, pp.24-31, September 2015.

[16] Chen KS, Wang CH, Tan KH, Chiu S F, "Developing one-sided specification six-sigma fuzzy quality index and testing model to measure the process performance of fuzzy information", International Journal of Production Economics, vol.208, pp.560-5, February 2019.

[17] Vojt J, "Deep neural networks and their implementation", 2016.

[18] Binu D andKariyappa B S, "RideNN: A new rider optimization algorithm-based neural network for fault diagnosis in analog circuits", IEEE Transactions on Instrumentation and Measurement, vol.68, no.1, pp.2-6, May 2018.

[19] Yi H, Shiyu S, Xiusheng D, Zhigang C, “A study on deep neural networks framework”, In proceedings of 2016 IEEE Advanced Information Management, Communicates, Electronic and Automation Control Conference (IMCEC), pp.1519-1522, 2016.

[20] Ekhool-Top learning management system from "https://ekhool.com/". 